\title{
Nasal nitric oxide is low early in life: case study of two infants with primary ciliary dyskinesia
}

\author{
E. Baraldi*, M.F. Pasquale*, A.M. Cangiotti", S. Zanconato*, F. Zacchello*
}

Nasal nitric oxide is low early in life: case study of two infants with primary ciliary dyskinesia. E. Baraldi, M.F. Pasquale, A.M. Cangiotti, S. Zanconato, F. Zacchello. (C) ERS Journals Ltd 2004.

ABSTRACT: Nasal nitric oxide levels are low in patients with primary ciliary dyskinesia, but it is not known whether this defect is already present in the first months of life.

The current authors measured nasal nitric oxide in two infants with situs inversus and primary ciliary dyskinesia, diagnosed by electron microscopy at 4 and 6 months of age, and in five healthy control infants.

Nasal nitric oxide values in the primary ciliary dyskinesia infants $(85$ and 115 parts per billion (ppb)) were markedly lower than in the healthy controls (mean: 295 ppb, range: $225-379 \mathrm{ppb})$.

This is the first report to show that nasal nitric oxide values are already low in early life in primary ciliary dyskinesia children, supporting the hypothesis that a reduced production of nasal nitric oxide is an intrinsic feature of this disease. The current authors suggest that the nasal nitric oxide test may be a useful, noninvasive method for screening young children for primary ciliary dyskinesia in clinical practice.

Eur Respir J 2004; 24: 881-883.
*Dept of Paediatrics, University of Padova, Padova, and ${ }^{\#}$ Electron Microscopy Unit, Umberto I Hospital, Institute of Normal Human Morphology, Marche Polytechnic University, Ancona, Italy.

Correspondence: E. Baraldi

Dept of Paediatrics

Via Giustiniani 3

35128 Padova

Italy

Fax: 39498213502

E-mail: baraldi@pediatria.unipd.it

Keywords: Nasal nitric oxide test primary ciliary dyskinesia

Received: January 42004

Accepted after revision: March 302004
Primary ciliary dyskinesia (PCD) is an autosomal recessive disorder characterised by functional and/or structural congenital cilia abnormalities with an underestimated incidence of one per 15,000 [1]. Nowadays, although the basic mechanism of the disease is known, the molecular genetics of PCD and the causes of the phenotypic variability remain to be explained. PCD is a clinically heterogeneous disease; neonatal patients may present with cough and/or respiratory distress, but there are age-related differences in presentation and diagnosis is often delayed until adulthood [1]. The usual clinical picture is one of recurrent upper and lower respiratory symptoms (rhinosinusitis, recurrent otitis, recurrent cough, sputum production and bronchiectasis) with mirror-image organ arrangement in 50\% of the children. PCD is underdiagnosed because there is no simple screening test, like the sweat test for cystic fibrosis (CF), and patients have to be referred to specialised centres for nasal brushing to estimate ciliary beat frequency and electron microscopy. The saccharine test, a noninvasive available method, is unsuitable for young children. Biopsy of the ciliated mucosa in the nose or bronchi is needed to confirm the diagnosis [1]. Early diagnosis is important in order to initiate rigorous treatment to prevent irreversible damage, such as bronchiectasis.

In 1994, very low levels of nasal nitric oxide (NO) in PCD children were first reported by LUNDBERG et al. [2], and were later confirmed by other groups in large numbers of children $[3,4]$. NO is thought to be involved in both ciliary motility regulation and host defence $[5,6]$, but the mechanism behind such a low nasal NO production in PCD is unknown. Articles published so far on nasal NO and PCD deal with school-aged children $[2-4,7]$, and it is not known whether there is a reduced nasal NO output already in the initial weeks of life, before the onset of upper airway symptoms.
In this case report, nasal NO was measured in two infants whose diagnosis of PCD was established in the first months of life.

\section{Case reports}

\section{Subjects}

Two infants with complete mirror-image organ arrangement (situs inversus) and PCD were evaluated. Patient A was a male aged 4 months (birth weight: $4,100 \mathrm{~g}$, gestational age: 42 weeks) with a history of unexpected respiratory distress at birth and persistent cough. Patient B was a female aged 6 months (birth weight: $2,900 \mathrm{~g}$, gestational age: 39 weeks) with a history of tachypnoea at birth, followed by three episodes of wheezing and cough in the first 4 months of life. PCD was diagnosed at 4 and 6 months of age, respectively, by bronchial brushing and ciliary morphology using electron microscopy $[1,8]$. Both children were clinically stable at the time of diagnosis, they had shown no evidence of respiratory exacerbation for at least 4 weeks and they were not taking any medication. The ciliary ultrastructural defect found in infant A was the absence of both outer and inner dynein arms; the outer dynein arms, when discernable, appeared short and stubby. Inner dynein-arms defect was prevalent in infant $\mathrm{B}$, frequently associated with central-pair displacement or absence, with or without transposition. The ultrastructural study was performed at the Electron Microscopy Unit, University of Ancona, Ancona, Italy.

The healthy group consisted of five healthy infants with no history of respiratory disease (mean age: 3 months and 24 days, range: 1.3-7 months). 


\section{Nasal nitric oxide measurement}

The nasal NO levels were recorded by inserting a nasal olive in one nostril, completely occluding the nostril to avoid ambient air sampling [9]. The olive was connected to the chemiluminescence analyser (CLD 77 AL Med; EcoPhysics, Durnten, Switzerland) via a Teflon tube. The contralateral nostril was left open. Nasal air was sampled continuously with a constant transnasal flow of $110 \mathrm{~mL} \cdot \mathrm{min}^{-1}$ for $\geqslant 30 \mathrm{~s}$. The test was repeated twice, recording the highest nasal NO value observed. Ambient NO levels were $<15$ parts per billion (ppb) during the measurement. Informed consent was obtained from the children's parents.

\section{Results}

The results are shown in figure 1 . The mean nasal NO value in healthy infants was $295 \mathrm{ppb}$ (range: 225-379), with individual values 2-3 times higher than the values found in the PCD patients ( 85 and $115 \mathrm{ppb}$ ). No overlap in nasal NO values was observed between the PCD infants and the healthy controls.

\section{Discussion}

Recent studies have demonstrated very low levels of nasal $\mathrm{NO}$ in patients with PCD and this measurement has been proposed as a useful diagnostic marker for screening suspected cases $[2,3,7,10]$. The current authors' finding of markedly lower levels of nasal NO in PCD children only a few months old is consistent with previous studies in older children, and supports the hypothesis that a reduced nasal NO production is an intrinsic feature of PCD, occurring early in life. To the current authors' knowledge, this is the first report showing low nasal NO values in infants with PCD, whereas other studies have been performed in older children and adults $[2-4,7,10]$.

The sensitivity and specificity of the nasal NO test in PCD have proved to be very good [7, 10], although a partial reduction of nasal NO levels can also be found in children with $\mathrm{CF}$ and sinusitis [7, 11]. In these latter diseases, reduced nasal NO values may be the consequence of impaired NO diffusion from the sinuses. Indeed, this mechanism could also explain the reduction in nasal NO values in symptomatic PCD, which invariably coincides with a chronic involvement

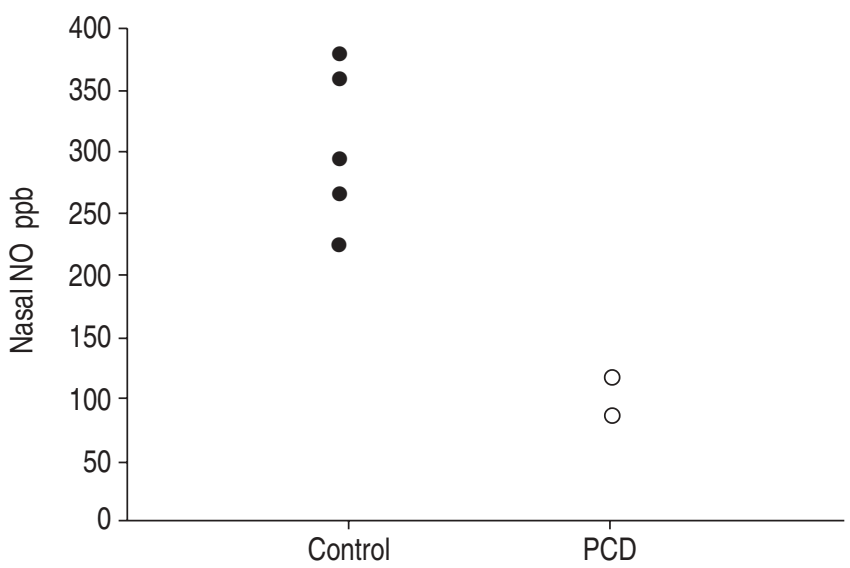

Fig. 1. - Nasal nitric oxide (NO) values measured in parts per billion $(\mathrm{ppb})$ in primary ciliary dyskinesia $(\mathrm{PCD})$ infants $(\bigcirc)$ and healthy control infants $(\mathbf{O})$ of the sinuses. However, an impaired NO diffusion is unlikely in the studied infants, because low nasal NO values were found even before the onset of recurrent upper respiratory symptoms.

The role of NO in the upper airways is not entirely clear. Although infants have poorly developed and only partially pneumatised paranasal sinuses, a considerable amount of nasal NO has been found in human newborns [12]. It has been suggested that nasal NO may be associated with host defence functions, including direct toxic effects on microorganisms and the regulation of mucociliary function $[5,6]$. As the majority of respiratory epithelial cells are ciliated and contain NO synthase, it may be that NO modulates airway ciliary beating [10].

At the present time, too many children with PCD are not being diagnosed early enough and are consequently not being treated appropriately $[1,13]$. Early diagnosis may prevent the onset of bronchiectasis and the subsequent decline in lung function $[1,13,14]$. Therefore, the nasal NO test may represent a simple method for screening for PCD [2, 3, 7], and an American Thoracic Society/European Respiratory Society group is working to establish guidelines on how to use this measurement in children suspected of having this disease. The method used in this study is feasible in infants and young children; however, larger studies are necessary to evaluate its sensitivity and specificity in noncooperative children. A possible limitation of the sampling method that the current authors have used is that air could be also collected from the lower airways. However, recording the highest nasal NO values and not the plateau, the current authors are confident that the nasal NO peak was not influenced by lower airways. The nasal NO values that were found in the two PCD infants are higher compared to values reported in older children [7]. This could be related to the disease duration and to the complications of PCD in older children, such as sinusitis, which is known to be associated with low nasal NO values.

In conclusion, these findings show that nasal nitric oxide values are already low at an early age in primary ciliary dyskinesia children, supporting the hypothesis that a reduced production of nasal nitric oxide is an intrinsic feature of this disease. Even though further studies are needed to standardise the method and to confirm these preliminary observations, the current authors suggest that the nasal nitric oxide test may be a useful, rapid and noninvasive method, feasible even in infants, for screening children for primary ciliary dyskinesia in clinical practice.

\section{References}

1. Bush A, Cole P, Hariri M, et al. Primary ciliary dyskinesia: diagnosis and standards of care. Eur Respir J 1998; 12: 982 988.

2. Lundberg JO, Weitzberg E, Nordvall SL, Kuylenstierna R Lundberg JM, Alving K. Primarily nasal origin of exhaled nitric oxide and absence in Kartagener's syndrome. Eur Respir J 1994; 7: 1501-1504.

3. Karadag B, James AJ, Gultekin E, Wilson NM, Bush A Nasal and lower airway level of nitric oxide in children with primary ciliary dyskinesia. Eur Respir J 1999; 13: 1402-1405.

4. Grasemann H, Gartig SS, Wiesemann HG, Teschler H, Konietzko N, Tatjen F. Effect of L-arginine infusion on airway NO in cystic fibrosis and primary ciliary dyskinesia syndrome. Eur Respir J 1999; 13: 114-118.

5. Jain B, Rubinstein I, Robbins RA, Sisson J. TNF- $\alpha$ and IL$1 \beta$ upregulate nitric oxide-dependent ciliary motility in bovine airway epithelium. Am J Physiol 1995; 268: L911L917.

6. Lundberg JO, Farkas-Szallasi T, Weitzberg E, et al. High 
nitric oxide production in human paranasal sinuses. Nature Med 1995; 1: 370-373.

7. Narang I, Ersu IR, Wilson NM, Bush A. Nitric oxide in chronic airway inflammation in children: diagnostic use and pathophysiological significance. Thorax 2002; 57: 586-589.

8. Pifferi M, Cangiotti AM, Ragazzo V, Baldini G, Cinti S, Boner AL. Primary ciliary dyskinesia: diagnosis in children with inconclusive ultrastructural evaluation. Pediatr Allergy Immunol 2001; 12: 274-282.

9. Baraldi E, de Jongste JC. ERS/ATS statement. Measurement of exhaled nitric oxide in children, 2001. Eur Respir J 2002; 20: 223-237.

10. Wodehouse T, Kharitonov SA, Mackay IS, Barnes PJ, Wilson R, Cole PJ. Nasal nitric oxide measurements for the screening of primary ciliary dyskinesia. Eur Respir $J$ 2003; 21: 43-47.

11. Baraldi E, Azzolin N, Biban P, Zacchello F. Effect of antibiotic therapy on nasal nitric oxide concentration in children with acute sinusitis. Am J Respir Crit Care Med 1997; 155: 1680-1683.

12. Shedin U, Norman M, Gustafsson L, Herin P, Frostell C. Endogenous nitric oxide in the upper airways of healthy newborn infants. Pediatr Res 1996; 40: 148-151.

13. Bush A, O'Callaghan C. Primary ciliary dyskinesia. Arch Dis Child 2002; 87: 363-365.

14. Ellerman A, Bisgaard H. Longitudinal study on lung function in a cohort of primary ciliary dyskinesia. Eur Respir J 1997; 10: 2376-2379. 